\title{
Superior Vena Cava Syndrome in Thoracic Malignancies
}

\author{
Philipp M Lepper MD, Sebastian R Ott MD, Hanno Hoppe MD, \\ Christian Schumann MD, Uz Stammberger MD, Antonio Bugalho MD, \\ Steffen Frese MD, Michael Schmücking MD, Norbert M Blumstein MD, \\ Nicolas Diehm MD, Robert Bals MD PhD, and Jürg Hamacher MD
}

\author{
Introduction \\ Anatomy and Physiology \\ Etiology \\ Infectious, Inflammatory, and Non-malignant SVCS \\ Malignancy \\ Clinical Manifestations \\ Assessment \\ Imaging \\ Biopsy and Staging \\ Management \\ General Treatment Considerations \\ Endovascular Treatment \\ Emergency External Beam Megavoltage Radiation Therapy \\ Chemotherapy and Immunotherapy \\ Steroids \\ Thromobolytics \\ Long-Term Anticoagulation \\ Endovascular and Surgical Interventions \\ Outcome \\ Summary
}

The superior vena cava syndrome (SVCS) comprises various symptoms due to occlusion of the SVC, which can be easily obstructed by pathological conditions (eg, lung cancer, due to the low internal venous pressure within rigid structures of the thorax [trachea, right bronchus, aorta]). The resulting increased venous pressure in the upper body may cause edema of the head, neck, and upper extremities, often associated with cyanosis, plethora, and distended subcutaneous vessels. Despite the often striking clinical presentation, SVCS itself is usually not a life-threatening condition. Currently, randomized controlled trials on many clinically important aspects of SVCS are lacking. This review gives an interdisciplinary overview of the pathophysiology, etiology, clinical manifestations, diagnosis, and treatment of malignant SVCS. Key words: superior vena cava syndrome; small-cell lung cancer; SCLC; non-small-cell lung cancer; NSCLC; lymphoma; venous stenting; emergency external beam radiation therapy; glucocorticoids. [Respir Care 2011;56(5):653-666. (C) 2011 Daedalus Enterprises] 


\section{Introduction}

Superior vena cava syndrome (SVCS) includes various clinical signs and symptoms due to external compression (or intrinsic obstruction) of the SVC itself or greater veins emptying into the SVC or the superior cavo-atrial junction, resulting in reduced blood flow. Clinical signs include cyanosis, plethora, distention of and missing positional emptying of subcutaneous vessels, and edema of the upper extremities, head, and neck. Edema may compromise the function of the larynx or pharynx, causing dyspnea, stridor, cough, hoarseness, and dysphagia. A more serious consequence is cerebral edema, which may cause headache, confusion, and, possibly, coma. Cough, dyspnea, and orthopnea are common symptoms and may mimic congestive heart failure or pericardial disease. However, sound physical examination should clarify the diagnosis in most patients.

The assessment of a patient with SVCS should be prompt and focus on the presence or absence of findings that suggest an elevated upper venous pressure in need of timely intervention. The spectrum of possible interventions in SVCS has increased from radiotherapy and chemotherapy to thrombolytic treatment and SVC stenting; the optimal treatment of choice should be decided with an interdisciplinary consensus. Though a general recommendation sup-

Philipp M Lepper MD, Robert Bals MD PhD, and Jürg Hamacher MD are affiliated with the Department of Internal Medicine V, University Hospital of Saarland, Homburg/Saar, Germany. Jürg Hamacher MD and Sebastian R Ott MD are affiliated with the Department of Pneumology, Universitätsspital Bern, University of Bern, Bern, Switzerland. Hanno Hoppe MD is affiliated with the Department of Radiology, Universitätsspital Bern, University of Bern, Bern, Switzerland. Christian Schumann MD is affiliated with the Division of Pulmonary Diseases, Department of Internal Medicine, University Hospital Ulm, Ulm, Germany. Uz Stammberger MD and Steffen Frese MD are affiliated with the Department of Thoracic Surgery, Universitätsspital Bern, University of Bern, Bern, Switzerland. Antonio Bugalho MD is affiliated with the Departamento de Pneumologia, Hospital de Pulido Valente, Faculdade de Ciências Médicas, Universidade Nova de Lisboa, Lisbon, Portugal. Michael Schmücking MD and Norbert M Blumstein MD are affiliated with the Department of Radiation Therapy, Universitätsspital Bern, University of Bern, Bern, Switzerland. Nicolas Diehm MD is affiliated with the Department of Clinical and Interventional Angiology, Universitätsspital Bern, University of Bern, Bern, Switzerland. Jürg Hamacher MD is also affiliated with Department of Pulmonary Medicine, Lindenhofspital, Bern, Switzerland.

Drs Lepper and Ott are co-first authors of this paper.

The authors have disclosed no conflicts of interest.

Correspondence: Philipp M Lepper MD, Department of Internal Medicine V, University Hospital of Saarland, D-66421 Homburg, Germany. E-mail: philipp.lepper@gmx.de.

DOI: $10.4187 /$ respcare.00947 porting the consideration of radiation therapy and/or stent placement for symptomatic SVC obstruction from lung cancer has been made by both the American College of Chest Physicians $^{1}$ and the National Comprehensive Cancer Network (http://www.nccn.org/professionals/physician_gls/ $\mathrm{PDF} / \mathrm{nscl}$.pdf), more detailed recommendations are currently lacking, as many aspects of SVCS do not have a good evidence base. As outlined by Rowell and Gleeson, ${ }^{2}$ there is a lack of well designed randomized controlled trials. Additionally, there is wide variation in the data and no standardized scoring system to measure both response and treatment-related morbidity. In all involved specialty fields there are technical innovations that may lead to patient benefit but that are not consistently studied in this specific clinical setting. We searched MedLine and the Cochrane Database to identify primarily randomized controlled trials on various aspects of SVCS. Of special interest were steroid treatment, oral or intravenous chemotherapy, megavoltage external beam radiation therapy, insertion of an expandable metal stent into the SVC (with or without thrombolysis or anticoagulation), or any combination of these treatments. We used the following terms (National Library of Medicine medical subject headings [MESH] identifier in brackets, if available): superior vena cava syndrome (D013479), vena cava obstruction, steroids (D013256), dexamethasone (D003907), prednisone, prednisolone (D011239), radiotherapy (D011882), radiation therapy (Q000532), stent, small-cell lung cancer (D018288), non-small-cell lung cancer (D002289), and combinations of those terms. We searched the database in June 2010 for randomized trials. We furthermore searched for observational studies on the specific aspects explored in the text, as well as those contributed by the involved subspecialists. Based on the heterogeneity of both etiology and therapeutic options in the context of pertinent clinical questions, major recent innovations in involved subspecialty fields, the striking sparseness of randomized clinical studies (even from national cancer centers and tertiary care facilities ${ }^{3}$ ), and the existence of some small meta-analyses, we aimed systematically to review the current literature up to June 2010. We decided against an additional metaanalysis.

\section{Anatomy and Physiology}

The SVC transports blood from the head and neck, upper extremities, and parts of the chest toward the heart. It carries approximately one third of the total venous return to the heart. SVC compression can be caused by tumor masses in the middle or anterior mediastinum, usually to the right of the midline. Cardiac output may be transiently diminished due to acute SVC obstruction, but within a few hours usually an increased venous pressure and collaterals achieve a novel steady state of blood return. Hemody- 
namic compromise is usually a result of mass effect on the heart rather than the SVC compression. ${ }^{4}$ Because of its relatively thin wall, compared to the aorta or trachea, and the low venous pressure, the SVC is among the first of the mediastinal structures to be obstructed. If the SVC becomes obstructed, blood flows through multiple smaller collaterals to the azygos vein or the inferior vena cava. These venous collaterals usually become dilated over several weeks, so that the upper body venous pressure is markedly elevated initially, but decreases over time..$^{5,6}$

\section{Etiology}

Mainly due to infectious disease (syphilis and tuberculosis) for several centuries, the primary cause of SVCS changed to malignancies some 25 years ago, and malignancies now account for up to $90 \%$ of all SVCS. ${ }^{7,8}$ In recent years thrombotic complications have increasingly occurred, reflecting the increased use of intravascular devices such as catheters, port-a-caths, pacemakers, and implantable defibrillators. ${ }^{9}$

\section{Infectious, Inflammatory, and Non-malignant SVCS}

In 1757, a Scottish physicist and anatomist, William Hunter, was the first to describe SVCS. He documented the findings of a 39-year-old man who died of a syphilitic aortic aneurysm. ${ }^{10}$ The 200 years since the first description were dominated by infectious etiologies of SVCS, mainly due to dilation of the aortic arch as a consequence of syphilitic involvement of the aorta. Until the middle of the 20th century, one third of cases were due to dilated aortic aneurysm, another third due to intrathoracic tumors, and the remainder were attributed predominantly to granulomatous mediastinal disease, most likely infectious mediastinitis from tuberculosis. ${ }^{11,12}$ Since the advent of antibiotics, however, the number of infectious cases of SVCS has considerably decreased.

Non-malignant conditions account for 15-40\% of SVC obstructions in contemporary retrospective series. ${ }^{8,9,13,14}$ Thrombosis of the SVC accounts for a substantial proportion of non-malignant causes of SVC obstruction. Most cases are related to the presence of indwelling intravascular devices. ${ }^{9,15}$ However, considering the increasing use of central venous catheters, the incidence of catheter-related SVC thrombosis appears to be low. ${ }^{16}$ Rarely, pulmonary embolism results from these thrombi, especially if they are attached to the catheter (sleeve thrombi) rather than the vessel wall (mural thrombi). ${ }^{17}$ Up to $50 \%$ of SVCS cases not due to malignancy are attributable to fibrosing mediastinitis, of which the most common cause is an excessive host response to a prior infection with Histoplasma capsulatum. Various other infections have been associated with fibrosing mediastinitis, including tuberculosis, acti- nomycosis, aspergillosis, blastomycosis, and Bancroftian filariasis. Infection with nocardiosis can cause SVCS by contiguous spread from a pulmonary, pleural, or cutaneous focus of infection. Post-radiation local vascular fibrosis should be included in the differential diagnosis of SVCS in patients who have received prior thoracic external beam radiation therapy, even if it predated the condition by several years. ${ }^{18}$ Additionally, vasculitic disorders (eg, Behçet disease) can also cause relevant venous occlusion. Table 1 summarizes non-malignant conditions that may lead to SVCS.

\section{Malignancy}

Intrathoracic malignancies are responsible for $60-85 \%$ of SVCS cases. SVC obstruction is the presenting symptom of a previously undiagnosed tumor in up to $60 \%$ of these cases. ${ }^{9}, 13,14$ Non-small-cell lung cancer (NSCLC) is the most common malignant cause of SVCS, accounting for $50 \%$ of all cases, followed by small-cell lung cancer (SCLC) (25\%) and non-Hodgkin lymphoma (10\%).9,14,19 Lung cancer and non-Hodgkin lymphoma account for approximately $95 \%$ of all malignant SVCS cases. ${ }^{9}$

Up to $4 \%$ of all lung cancer patients develop SVCS in the course of the lung cancer. SVCS occurs in up to $10 \%$ of SCLC patients. That difference is probably due to the different growth kinetics of SCLC and NSCLC, and the more frequent central localization of SCLC, compared to NSCLC. SVCS in lung cancer can occur if the primary tumor obstructs the vessel or due to enlarged mediastinal lymph nodes. Also, direct invasion of the SVC has been described, mostly in SCLC.

Up to $4 \%$ of all non-Hodgkin lymphoma patients develop SVCS. The incidence of SVCS varies considerably in non-Hodgkin lymphoma and depends on subtype. The highest incidence, almost $60 \%$, is found in patients with primary mediastinal large B-cell lymphoma with sclerosis, an unusual and aggressive non-Hodgkin lymphoma subtype (up to $7 \%$ of all diffuse large-cell lymphomas). ${ }^{20}$ Diffuse large-cell and lymphoblastic lymphomas are the most common subtype of non-Hodgkin lymphoma associated with SVCS. The incidence in these non-Hodgkin lymphoma types is up to $21 \%$, whereas the incidence in other non-Hodgkin lymphoma subtypes is approximately $4 \% .{ }^{21}$ In patients with intravascular lymphoma, intravascular obstruction can be the primary mechanism of SVCS. Interestingly, Hodgkin's lymphoma rarely causes SVCS, despite the fact that these patients commonly present with mediastinal lymphadenopathy. Other malignant entities, such as mesothelioma, teratoma, primary mediastinal germ cell tumors, and mediastinal lymph node metastases of other solid tumors, are less frequently associated with SVCS. 
Table 1. Differential Diagnoses of Malignant Superior Vena Cava Syndrome

\begin{tabular}{|c|c|c|}
\hline Condition & Incidence & Recommended Therapy and Notes \\
\hline Fibrosing mediastinitis & From rarity up to $50 \%$ & $\begin{array}{l}\text { Medical management of symptoms, conservative interventions } \\
\text { (dilation and stent implantation for stenoses), surgical } \\
\text { treatment of adhesions and strictures. } \\
\text { Treatment with methylsergide-maleate } \\
\text { Pentoxifylline plus tocopherol, amifostine, and hyperbaric } \\
\text { oxygen are somewhat experimental. } \\
\text { Antibiotics }\end{array}$ \\
\hline Histoplasmosis & Rather rare & \multirow{8}{*}{$\begin{array}{l}\text { Largely depends on sociocultural context; slow evolution, } \\
\text { therefore causing few symptoms; usually younger than } \\
\text { patients with malignancy, particularly in North America. } \\
\text { Especially tuberculosis. Alternatively causes mediastinal } \\
\text { structure obstructions by large lymph nodes. }\end{array}$} \\
\hline Tuberculosis & Rare & \\
\hline Actinomycosis & Rare & \\
\hline Aspergillosis & Rare & \\
\hline Blastomycosis & Rare & \\
\hline Bancroftian filariasis & Rare & \\
\hline Behçet disease & Rare & \\
\hline Rheumatic fever & Very rare $(<1 \%)$ & \\
\hline $\begin{array}{l}\text { Other: silicosis; sarcoidosis; familial multifocal } \\
\text { fibrosis; isolated aneurysms or ruptures of } \\
\text { the innominate artery; benign mediastinal }\end{array}$ & Very rare $(<1 \%)$ & $\begin{array}{l}\text { Radiofrequency ablation for atrial fibrillation more frequently } \\
\text { causes pulmonary artery stenosis. }\end{array}$ \\
\hline
\end{tabular}

tumors; after radiofrequency ablation

Aortic aneurysm; isolated aneurysms or ruptures of the innominate artery or other vascular abnormalities

\section{Clinical Manifestations}

The clinical appearance of SVCS is mainly determined by increased venous pressure in the upper body from the SVC obstruction. Normally, the cervical venous pressure range is $2-8 \mathrm{~mm} \mathrm{Hg}$. However, the limited patency of the SVC may increase the pressure up to 10 -fold (20$40 \mathrm{~mm} \mathrm{Hg}$ ) and thus causes an abnormally elevated hydrostatic pressure ${ }^{22-24}$ that causes edema of the head, neck, eyelids, upper torso, and arms, and visibly dilated veins, which is the most predominant symptom in SVCS. Characteristically, they are non-collapsing if above the heart level. Consecutive signs of decreased venous flow are cyanosis and plethora. Furthermore, edema of the larynx or pharynx may adversely affect their function and lead to complaints including hoarseness, cough, stridor, dyspnea, and dysphagia. Figure 1 shows the most frequent signs and symptoms in SVCS.

Patients with SVCS can, rarely, develop life-threatening complications such as cerebral edema, causing headache, dizziness, confusion, and eventually coma or compromised hemodynamics, if SVC obstruction impairs venous return to the right atrium. Hemodynamic alterations can also be caused by direct compression of the heart by a mediastinal mass. Blurred vision or conjunctival suffusion may indicate imminent papilledema. Another rare complication of SVCS is esophageal varices, which occasionally cause variceal bleeding, especially in chronic SVCS.

As a consequence of the increased cervical venous pressure, subcutaneous vessels may distend, providing collateral circulation to the lower torso and finally to the inferior vena cava. The most important collateral pathway to the inferior vena cava is via the azygos/hemiazygos veins and intercostal veins. Other collateral pathways include the internal mammary veins and their tributaries and conjunctions to the superior and inferior epigastric veins, and the long thoracic veins, which discharge into the femoral and vertebral veins. Unfortunately, it requires several weeks to develop a sufficient collateral vascular network, so it stands to reason that clinical manifestations of SVCS may vary and do not exclusively depend on the degree of vascular obstruction, but also on the rapidity of onset. Approxi- 


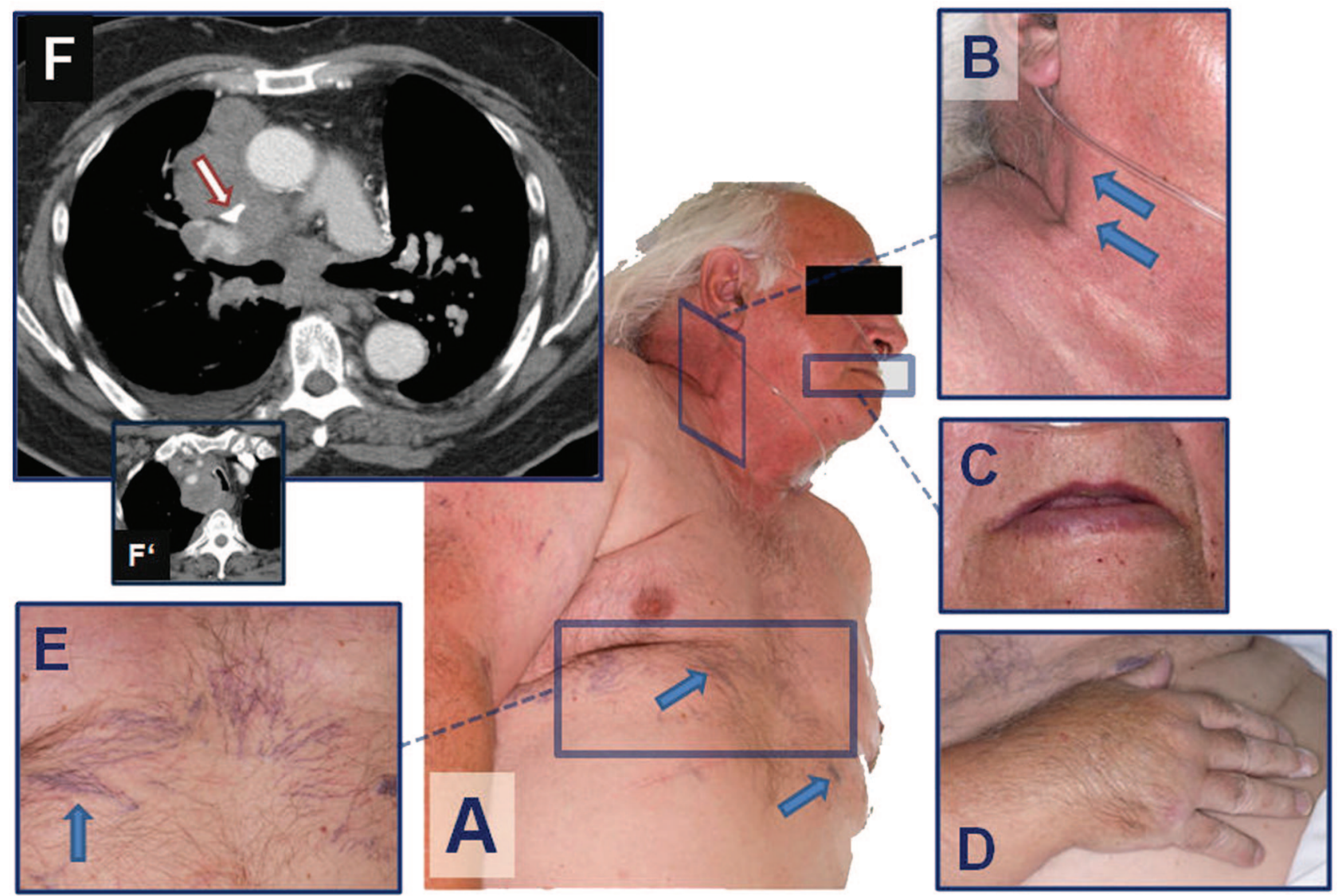

Fig. 1. Typical clinical findings in a patient with superior vena cava syndrome due to small-cell lung cancer. This patient presented with dyspnea and elevated $\mathrm{P}_{\mathrm{acO}}$. A: Plethora of face and neck. B: Distended jugular veins. C: Cyanosis of the lips. D: Right arm and hand massively swollen. E: Substantial collateral circulation (arrow). F: Computed tomogram shows compression of the superior vena cava (arrow) due to a large mediastinal mass, causing $\left(\mathrm{F}^{\prime}\right)$ tracheal compression and deviation, and stridor.

mately one third of all SVCS patients develop clinical signs and symptoms of SVC obstruction within a 2-week period, but sometimes it can take longer.

In summary, facial edema, dilated head, neck, and chest veins, dyspnea, cough, and in some cases, orthopnea are the most common symptoms of SVCS. Although the clinical appearance of SVCS can be striking at times (see Fig. 1) and the patient may appear severely distressed, SVCS itself is rarely fatal. Only when severe cerebral edema or hemodynamic alterations complicate the course of SVCS is a patient at high risk of dying.

\section{Assessment}

Usually, the clinical diagnosis of SVCS is based on a quite clear clinical presentation, with a combination of the aforementioned signs and symptoms. A careful physical examination can rule out common differential diagnoses that may mimic SVCS, including congestive heart failure and Cushing syndrome (eg, by ectopic production of adrenocorticotropic hormone). Physical examination should include a careful evaluation of central nervous functions, because neurologic alteration may be subtle, although already indicating imminent cerebral edema and, thus, a life-threatening complication and the need for immediate therapeutic intervention. Additionally, appraisal of respiratory function and hemodynamics is needed to determine the patient's risk of adverse outcome. Taking a detailed history of the current medical condition is equally important, and it should include onset and duration of symptoms and medical history, with emphasis on malignant conditions and recent intravascular procedures, including central venous lines.

Generally, symptoms develop over a period of several weeks, and their severity increases with duration of SVC obstruction. Of interest, formation of venous collaterals may improve clinical manifestations of SVCS in some cases.

In conclusion, patient history and a close physical examination constitute a solid basis for adequate management of patients with SVCS. The severity of symptoms determines the required diagnostic procedures and the 
Table 2. Signs and Symptoms of Superior Vena Cava Syndrome

\begin{tabular}{lcc}
\hline \hline \multicolumn{1}{c}{ Sign or Symptom } & $\begin{array}{c}\text { Estimated } \\
\text { Incidence } \\
\% \text { (range) }\end{array}$ & Critical Issue \\
\hline Hemodynamic & & \\
Facial edema & $82(60-100)$ & Hemodynamic \\
Arm edema & $46(14-75)$ & compromise \\
Distended neck veins & $63(27-86)$ & \\
Distended chest veins & $53(38-67)$ & \\
Facial plethora & $20(13-23)$ & \\
Visual symptoms & $2(\mathrm{ND})$ & \\
Hypotension & ND & \\
Respiratory & & \\
Dyspnea & $54(23-74)$ & Laryngeal edema \\
Cough & $54(38-70)$ & (relevant stridor) \\
Hoarseness & 17 (ND) & \\
Stridor & $4(\mathrm{ND})$ & \\
Neurologic & & \\
Syncope & $10(8-13)$ & Cerebral edema \\
Headaches & $9(6-11)$ & \\
Dizziness & $6(2-10)$ & \\
Confusion, obtundation, stroke & $6(\mathrm{ND})$ & \\
Other & & \\
Dysphagia & $\mathrm{ND}$ & \\
Cyanosis & $\mathrm{ND}$ & \\
\hline ND $=$ no data available & & \\
(Adapted from Reference 23.) & & \\
\hline
\end{tabular}

acuteness of therapeutic interventions. According to $\mathrm{Yu}$ and colleagues, ${ }^{25}$ hemodynamic symptoms (facial swelling) are most frequent, followed by respiratory symptoms (dyspnea, cough). Common symptoms are displayed in Table 2.

\section{Imaging}

The diagnosis of a large mediastinal mass can be made on a conventional chest radiograph. For a more detailed visualization of the SVC and its surrounding structures, a chest computed tomogram (CT) with intravenous contrast medium in the venous phase is recommended. Alternatively, magnetic resonance imaging (MRI) with MRI phlebocavography can be performed. Both CT and MRI can be used to diagnose the underlying pathology, including tumor mass size and localization. SVC diameter and length of SVC stenosis/occlusion can be determined, which constitutes a solid basis for planning endovascular treatment. Alternatively, a conventional phlebocavography with simultaneous intravenous contrast injection from both upper extremities can be performed. Since this is usually done in the angiography suite, venous pressure gradient measurements and stenting can be performed at the same time. The radiologic stages of vena cava obstruction, as suggested by Stanford and colleagues, ${ }^{26}$ are displayed in Figure 2.
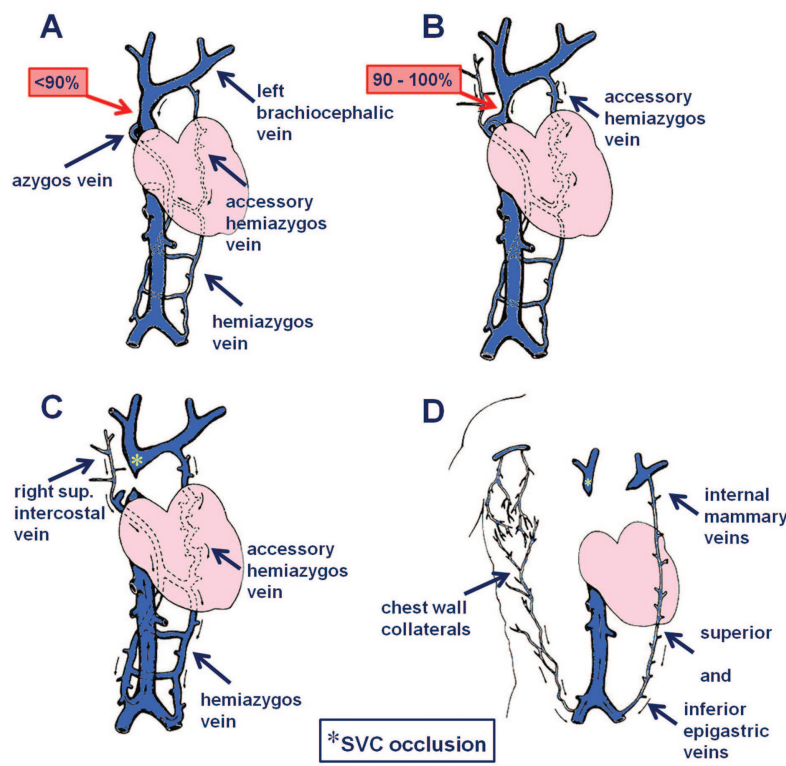

Fig. 2. Radiologic stages of vena cava obstruction. The Stanford classification system for superior vena cava (SVC) syndrome was developed to assist in identifying patients at risk of substantial airway or cerebral compromise and therefore warranting rapid intervention. A: Stanford type I: mild SVC obstruction, with vessel obstruction of less than 90\%. B: Stanford type II: high-grade SVC stenosis (grade of stenosis 90-100\%). C: Stanford type III: complete SVC obstruction and prominent flow through collateral veins, but without involvement of the mammary and epigastric veins. D: Stanford type IV: complete SVC obstruction and prominent flow through collateral veins and the mammary and epigastric veins. (Adapted from Reference 26, with permission).

\section{Biopsy and Staging}

The treatment of SVCS is determined by the underlying disease, so the cornerstone of the treatment of thoracic malignancies is accurate diagnosis, and in the case of malignancy an exact determination of tumor extension and stage. In lung cancer, the presence of SVCS usually indicates an extensive mediastinal tumor mass. In addition to morphological imaging modalities such as CT or MRI, positron emission tomography (a molecular imaging modality), provides further information on node status and mediastinal involvement. Additionally, a cranial CT or preferably a cranial MRI and, if necessary, a bone scintigraphy ${ }^{27}$ may complete the staging procedures. However, to clarify the nature of the underlying disease, biopsies have to be obtained for histological and/or cytological examination.

A diagnostic bronchoscopy, either in flexible or rigid technique, can detect endoluminal tumor growth, as well as tumor infiltration of central and peripheral airways, and obtain tumor tissue samples, via forceps or flexible cryoprobe. ${ }^{28}$ In addition, cytological methods such as protected specimen brush, bronchial washing, or bronchoalveolar lavage tend to increase the diagnostic yield. 


\section{Superior Vena Cava Syndrome in Thoracic Malignancies}

Table 3. Grading of Superior Vena Cava Syndrome

\begin{tabular}{|c|c|c|c|}
\hline Grade & Severity & Estimated Incidence $(\%)$ & Definition \\
\hline 0 & Asymptomatic & 10 & $\begin{array}{l}\text { Radiographic evidence of superior vena cava obstruction in the absence of signs and } \\
\text { symptoms (see Figure 3) }\end{array}$ \\
\hline 1 & Mild & 25 & Edema of head or neck (vascular distension), cyanosis, plethora \\
\hline 2 & Moderate & 50 & $\begin{array}{l}\text { Edema of head or neck, with functional impairment (mild dysphagia, cough, mild or } \\
\text { moderate impairment of head, jaw, or eyelid movement, visual disturbances due to } \\
\text { eyelid edema) }\end{array}$ \\
\hline $3 *$ & Severe & 10 & $\begin{array}{l}\text { Mild or moderate cerebral edema (headache, dizziness) or mild to moderate laryngeal } \\
\text { edema or diminished cardiac reserve (syncope after bending) }\end{array}$ \\
\hline $4^{*}$ & Life-threatening & 5 & $\begin{array}{l}\text { Substantial cerebral edema (confusion, obtundation) or substantial laryngeal edema } \\
\text { (stridor) or substantial hemodynamic compromise (syncope without precipitating factors, } \\
\text { hypotension, renal insufficiency) }\end{array}$ \\
\hline $5^{*}$ & Fatal & $<1$ & Death \\
\hline
\end{tabular}

Conventional mediastinoscopy is limited to paratracheal lymph nodes ( $2 \mathrm{R}, 2 \mathrm{~L}, 4 \mathrm{R}$, and $4 \mathrm{~L}$ ), pretracheal nodes (stations 1 and 3), and anterior subcarinal nodes (station 7). Newer techniques such as video-assisted mediastinoscopic lymphadenectomy allow better visualization and more extensive sampling. ${ }^{29}$ Even if a complete mediastinal lymphadenectomy is performed, not all lymph nodes can be assessed. To avoid bleeding complications in SVCS, less invasive diagnostic methods are preferred.

Mediastinal lymph nodes can be biopsied under conventional anatomical or CT-based localization or newer imaging methods. Endobronchial ultrasound guided fineneedle aspiration biopsy of mediastinal lymph nodes has a high diagnostic accuracy and offers an alternative and less invasive technique for biopsy of mediastinal lymph nodes, as compared to mediastinoscopy. ${ }^{30}$ Endobronchial ultrasound can overcome the limitations of mediastinoscopy in assessing lymph node stations $2,3,4,7,10$, and 11, and is preferred in terms of safety and costs.

Sputum cytology, pleural fluid cytology, and biopsy of peripheral enlarged lymph nodes (eg, supraclavicular) are recommended if these are present and might be diagnostic in up to two thirds of cases. ${ }^{31}$

\section{Management}

Treatment of the underlying disease strongly depends on histology type, staging of the disease, previous treatments, and overall prognosis. Novel molecular biological targets such as epidermal growth factor receptor tend to give further important information on treatment options. If, after obtaining medical history, clinical assessment, and chest imaging, malignant SVCS is probable, management depends on the presence or absence of grade 4 symptoms (ie, critical issues for the patient, such as threatened air- way, cardiac compression, and/or hypotension or syncope without preceding factors) (Table 3 ). If the patient presents with grade 4 symptoms, venogram and urgent stenting should be considered. If thrombosis is present, the patient might benefit directly from thrombolytic agents. A threatened airway should immediately be protected. All other stages prompt tissue biopsy and staging, which should be done without relevant delay. Early multidisciplinary planning improves the management of SVCS. If neurologic symptoms are present, brain metastases should be excluded with imaging (preferably contrast-enhanced MRI, alternatively contrast-enhanced CT). After biopsy and staging, a tumor-specific and stage-specific treatment plan should be developed depending on the tumor entity and symptoms, and, in the case of malignancy, on the Eastern Cooperative Oncology Group (ECOG) Performance Status Scale score (http://ecog.dfci.harvard.edu/general/perf_stat.html). Figure 3 summarizes a treatment algorithm for the management of malignant SVCS.

Although the prognosis of most malignancies causing SVCS is poor, most of the underlying conditions may respond well to chemotherapy, radiation therapy or combined chemotherapy and radiation therapy; several months of survival can be seen in properly treated patients. As SVCS might be the first manifestation of a thoracic malignancy, even in advanced-stage malignancies all therapeutic options, including invasive ventilation, should be considered, as they might gain several months of survival.

\section{General Treatment Considerations}

The primary goal in SVCS management is alleviation of symptoms and treatment of the underlying disease. We recommend that the patient's head be raised to decrease hydrostatic pressure and head and neck edema, though this 


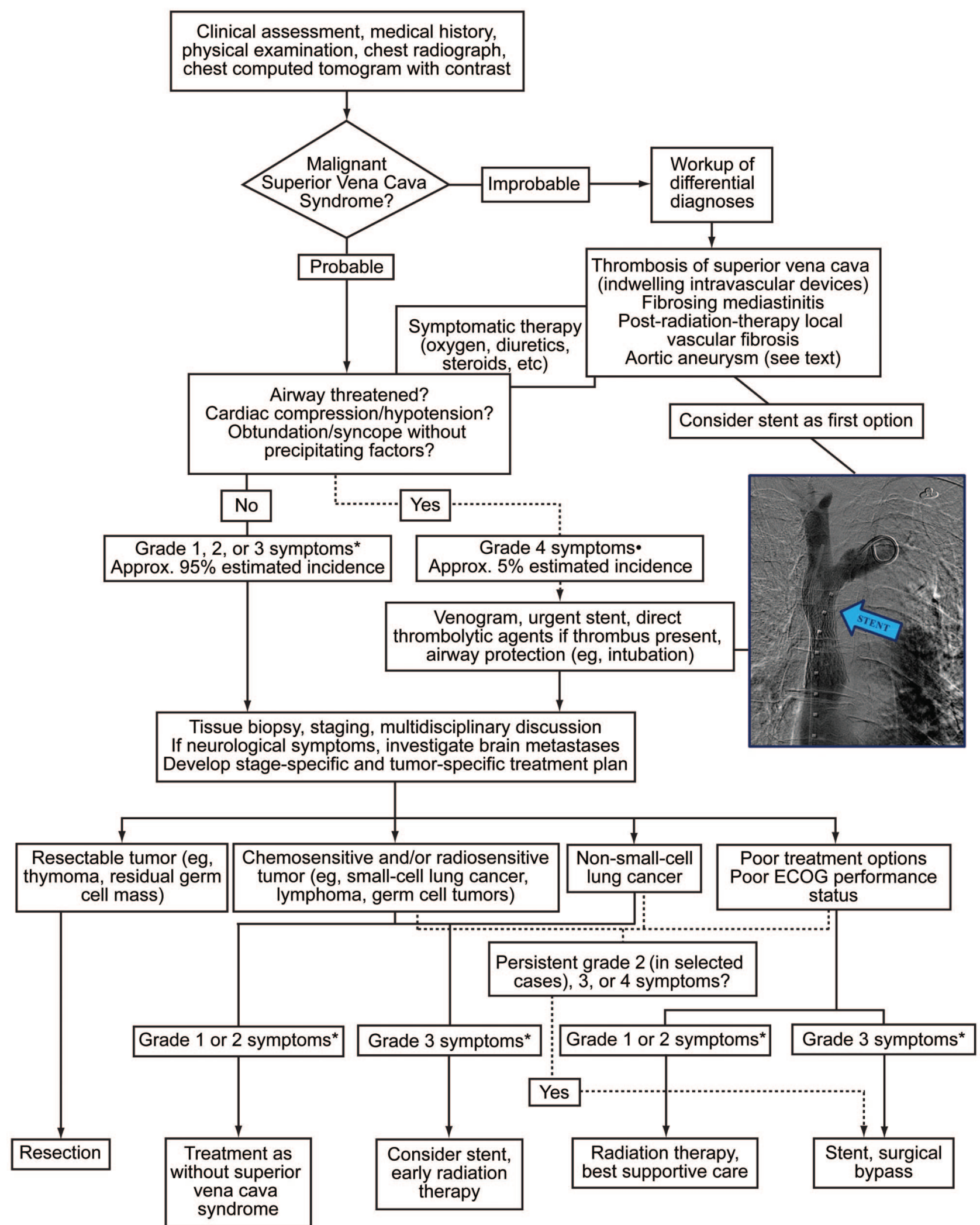

Fig. 3. Management algorithm for superior vena cava syndrome (SVCS). ${ }^{*}$ For grading see Table 3. ECOG = Eastern Cooperative Oncology Group Performance Status Scale. 
approach is not backed by data. Obstruction of blood flow through the SVC slows venous return, which can cause local irritation or thrombosis of veins in the upper extremities and delayed absorption of drugs from the surrounding tissues, so intramuscular and intravenous injections in the arms should be avoided. If SVCS results from an intravascular thrombus associated with an indwelling catheter, removal of the catheter and systemic anticoagulation are indicated.

Steroids (see below) are recommended only in patients with steroid-sensitive tumors, and in patients undergoing external beam radiation therapy. The use of diuretics is a matter of debate. Diuretics are recommended, although it is unclear whether small changes in right atrial pressure affect venous pressure distal to the obstruction. As the safety profile of diuretics is good and diuretics are commonly well tolerated, their use in SVCS is justified. However, if diuretics do not alter symptoms, they should be stopped.

\section{Endovascular Treatment}

The majority of patients with malignant SVCS are under palliative care, which is associated with survival of approximately 6 months. ${ }^{32}$ Since SVCS symptoms may be quite distressing, timely palliation is indicated in most of these patients. Treatment modalities include SVC stenting, irradiation, chemotherapy, and bypass surgery. However, bypass surgery is quite invasive for a palliative procedure, and irradiation may not be feasible if cumulative maximum dose has been reached in previous treatments. Furthermore, irradiation or chemotherapy may take days to weeks to become clinically effective. ${ }^{33}$

SVC stenting (Fig. 4) is effective and has few complications, $^{34-36}$ so many institutions, including ours, prefer primary stenting in de novo SVCS and do not restrict stenting to patients with recurrent SVCS. ${ }^{37}$ In most of our patients, stenting has markedly improved quality of life, and patients were recurrence-free prior to exitus from the underlying malignancy.

Endovascular stenting is usually performed with a femoral approach, sometimes combined with a jugular or subclavian approach, using an 9-F sheath. Usually an intravenous bolus of heparin $(5,000$ international units) is administered at the onset of the procedure. The SVC stenosis or occlusion is passed with a steerable hydrophilic guidewire (eg, Terumo, Tokyo, Japan) and a selective catheter (headhunter or multipurpose tip configuration). The hydrophilic wire is then exchanged for a stiff working wire (eg, Amplatz, Cook Medical, Bloomington, Indiana) and the catheter is removed. Subsequently, a self-expanding stent (eg, Sinus XXL, Optimed, Ettlingen, Germany; or Wallstent, Boston Scientific, Natick, Massachusetts; or Gianturco-Z, Cook Medical, Bloomington, Indiana) is placed,

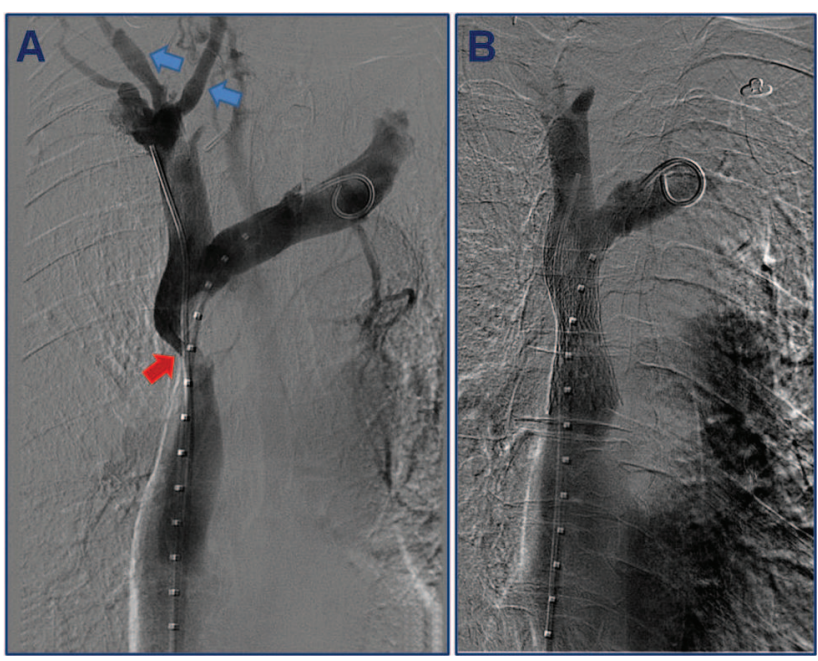

Fig. 4. Angiography shows superior vena cava (SVC) stenting in a 55-year-old woman with small-cell lung cancer and Stanford type II SVC obstruction on admission. A: Lumen narrowing of $>90 \%$ (red arrow). Note the congestive dilation of veins draining into the SVC (blue arrows), which disappeared immediately after stent placement. B: After placement of a $22 \times 60 \mathrm{~mm}$ Sinus XXL stent in the SVC vessel patency was restored completely.

with oversizing of approximately $10 \%$ relative to the unaffected SVC diameter, to prevent migration, especially toward the right atrium. If necessary, careful dilation of the stent with an angioplasty balloon can be performed. In the case of a short stenosis at a location where stent migration may cause a problem, a balloon expanding stent may be used (eg, Palmaz or Strecker stent).

In most cases, unilateral stent placement is sufficient. However, under certain circumstances bilateral stent placement in both brachiocephalic veins and the SVC may be necessary. At our institution we compared the results of 61 unilateral stent placements to 22 bilateral stent placements. ${ }^{37}$ Technical success was achieved in $99 \%$ of patients and clinical success in $91 \%$ of patients in the unilateral group, and $90 \%$ of patients in the bilateral group, which is similar to other reports. ${ }^{38}$ The occlusion rate was significantly lower in the unilateral group than in the bilateral group. The complication rate was higher in the bilateral group (28\%) than in the unilateral group (9\%). Stent patency tended to be longer in the unilateral group, but the difference was not significant. We concluded that both unilateral and bilateral stent placement are highly effective and expeditious in alleviating SVCS symptoms, but stent occlusion tended to occur more frequently after bilateral stenting. Unilateral SVC stenting was clinically effective with lower cost, easier placement, shorter procedure time, and lower rates of complications and recurrence.

Complications of SVC stenting are rather rare (between less than $4 \%$ and up to $28 \%$, and seem usually to be clearly less than 10\%), but may be fatal, including pericardial 
tamponade, pulmonary embolism, or stent migration to the right heart or pulmonary vasculature. ${ }^{39}$ Basically, anticoagulation is recommended after SVC stenting, which might interfere with necessary invasive diagnostic procedures if not done before, but duration and type of anticoagulation remain controversial. However, thrombolytics increased the complication rate after SVC stenting to as high as $10 \%$. The complications were related to bleeding problems such as cerebral, pulmonary, or pericardial hemorrhage with tamponade. 40

\section{Emergency External Beam Megavoltage Radiation Therapy}

Tumors are usually more or less radiosensitive, as described by the corresponding cell survival curves, the relationship between the absorbed radiation dose and the proportion of cells that "survive" in the sense that they are able to grow in a colony, thereby demonstrating retainment of their reproductive integrity. Before the era of endovascular stenting, radiation therapy as a sole treatment was widely recommended in patients with SVCS. Prerequisite was no previous irradiation. With the advent of new systemic cancer drugs and the accumulation of radiobiological and clinical data from randomized phase III studies, it seems advisable to use concurrent chemoradiation therapy for epithelial tumors, to maximize tumor response. However, the patient's clinical condition has to be good enough to allow this treatment approach. The rationale for combined chemotherapy plus radiation therapy is as follows: due to the idiotopic effect of concurrent chemoradiation therapy, and due to shorter overall treatment time, accelerated repopulation of surviving clonogenic cells in tumors during the course of treatment is reduced. This approach seems to have the best chance to eradicate all clonogenic cells and provide the best local tumor control and patient survival. ${ }^{41-44}$ For epithelial tumors, concurrent chemoradiation therapy seems superior to sequential chemotherapy followed by radiation therapy, as demonstrated in several studies. So far, little evidence suggests that the addition of induction chemotherapy before irradiation confers any benefit for local-regional tumor control.43,45-47 One reason is that chemotherapy alone, like radiation therapy alone, may accelerate the proliferation of tumor cell clonogens. Another possibility is that induction chemotherapy may select for or induce drug-resistant cells that might be cross-resistant to radiation. Finally, chemotherapy drugs may, under certain conditions, increase the metastatic potential of tumor cells or damage normal tissues in such a way as to make them prone to metastatic growth. In SCLC patients, radiation alone or concurrent chemoradiation therapy may improve SVCS in up to $80 \%$ of patients. ${ }^{48,49}$ In certain settings, even for certain non-Hodgkin lymphoma subtypes, concurrent chemoradiation therapy might provide better local control than a sequential approach, and may therefore improve long-term survival. ${ }^{42}$ After SVC stenting in a patient with a malignancy, concurrent radiation therapy plus chemotherapy is usually advised to optimize outcome and prevent proximal and/or distal tumor growth in the stent (tertiary prevention).

Radiation may improve symptoms. In a retrospective analysis of 35 patients, 30 patients had reduced symptoms within 5-9 days, ${ }^{50}$ usually prior to radiologic improvement. This might be a consequence of augmented use of collaterals. Symptomatic patients should be referred to suitable, normally tertiary-care hospitals.

\section{Chemotherapy and Immunotherapy}

In patients presenting with non-Hodgkin lymphoma or germ-cell tumors, chemotherapy seems to be the treatment of choice for SVCS and may provide fast relief, as these tumors are exquisitely chemo-sensitive. In patients with SCLC, SVCS symptom relief from radiation or chemotherapy alone is equally effective. ${ }^{51}$ Using chemotherapy as the only treatment for these histologies, symptoms usually improve within 1-2 weeks of treatment initiation.

Adjuvant chemotherapy (ie, chemotherapy delivered after radiation therapy) may not improve outcome, so radiation therapy prior to chemotherapy might reduce chemotherapy efficacy, impede SVCS symptom relief, and cause poorer long-term results, ${ }^{21,52}$ which should be taken into account in the initial treatment decision.

Therapy can be individualized and targeted if molecular analysis of tumor tissue is available. For instance, the combination of radiation therapy with cetuximab might be effective, since overexpression of epidermal growth factor receptor reduces radiosensitivity, and radiation therapy may up-regulate the epidermal growth factor receptor. ${ }^{53,54} \mathrm{How}$ ever, so far there are no data on targeted therapies in the treatment of SVCS. Figure 5 shows an example of the benefits of multimodal therapy in SCLC.

\section{Steroids}

Glucocorticoids can be effective in steroid-sensitive malignancies such as lymphoma or thymoma, but their effectiveness has not been studied in malignancies considered non-steroid-sensitive, such as NSCLC, so it not clear whether steroids have a role in the management of acute SVCS. Steroids should therefore be used only in selected patients.

If the patient undergoes radiation therapy, steroids are commonly used to prevent post-radiation swelling. Especially in patients with preexisting laryngeal edema, steroids might be justified, though their effectiveness in this setting has not been formally studied in a randomized controlled trial. 

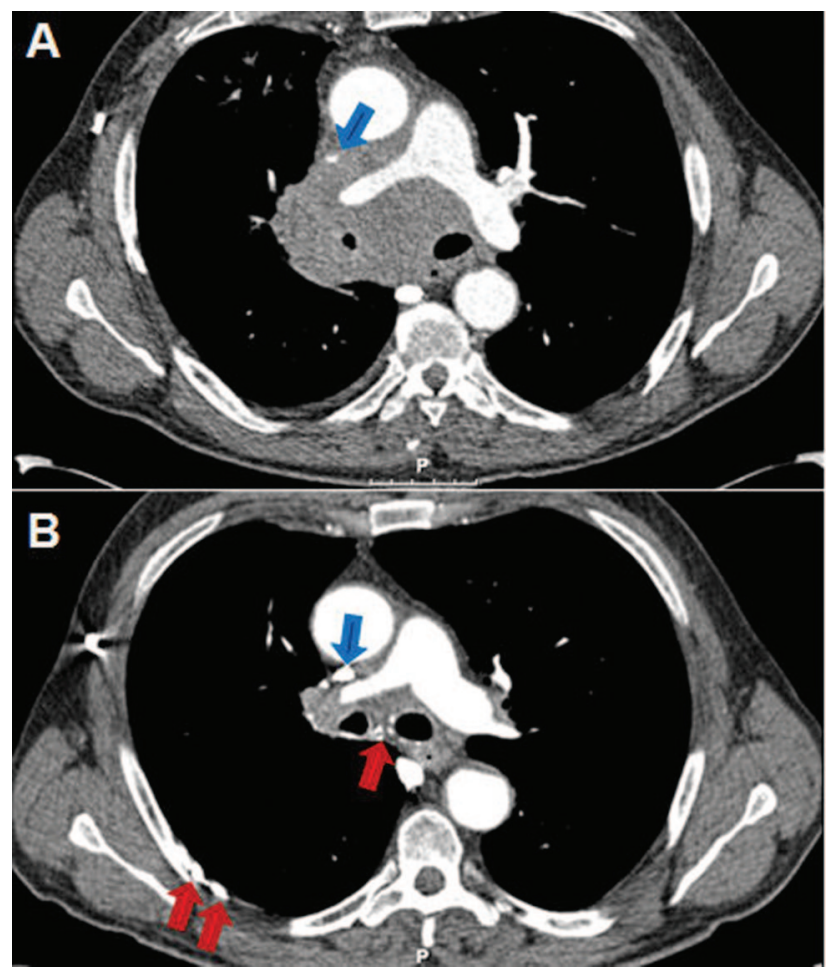

Fig. 5. Resolution of superior vena cava syndrome before $(A)$ and after (B) combined radiotherapy and chemotherapy in a 66-yearold patient with extensive small-cell lung cancer diagnosed in November 2008, treated with 4 cycles of cisplatin and etoposide and mediastinal radiotherapy (39 Gy). Initial therapy led to complete remission and a progression-free survival of 3 months. Survival was 14 months as of January 2010. SVCS patency is restored after combined therapy (blue arrows). Note collaterals (red arrows), which mostly developed after therapy.

\section{Thrombolytics}

Patients with extensive thrombosis in conjunction with stenotic SVC obstruction may benefit from local catheterdirected thrombolysis ${ }^{1,55}$ to reduce the length of the stented segment and the risk of embolization. Alternatively, the thrombus burden may be removed via mechanical endovascular thrombectomy. Adjunctive thrombolytic therapy after SVC stenting, however, did not improve primary patency but did increase the risk of bleeding, and is therefore not generally recommended. ${ }^{56}$ All patients with central venous thrombosis should receive anticoagulants for 3-6 months, because pulmonary emboli may result.

\section{Long-Term Anticoagulation}

Short-term anticoagulation is usually recommended for patients undergoing SVC stenting, 55,57 but it remains unclear whether SVCS patients really benefit from long-term anticoagulation. Full anticoagulation is reasonable after thrombolytic or mechanical removal of additional throm-

botic lesions. Whether low-molecular-weight heparin is superior to oral anticoagulation in this setting ${ }^{58}$ has not been prospectively addressed, nor has the question of whether patients who undergo SVC stenting require anticoagulation or dual antiplatelet therapy.

One possible clinical problem is heparin-related complications such as heparin-induced thrombosis. It is therefore important to monitor platelet count, and vigilance is needed if a rapid decline in platelets occur, as this suggests the possibility of platelet-induced thrombocytopenia (white clot syndrome), which may lead to life-threatening vascular occlusions and venous thromboemboli. ${ }^{40}$

\section{Endovascular and Surgical Interventions}

Although surgical intervention to bypass SVC obstruction is effective and associated with relatively few complications, its use has been widely replaced by endovascular treatments. Endovascular methods are effective as a "bail-out" strategy in patients with grade 4 symptoms and usually obviate surgical interventions in patients with limited life expectancy. In patients with malignant thymoma and thymic carcinoma, surgery should be evaluated as part of a multimodal treatment strategy. ${ }^{59,60}$ Patients with NSCLC up to Union for International Cancer Control clinical stage IIIA should be radically resected, if possible combined with adjuvant chemotherapy and with or without radiotherapy. ${ }^{61,62}$ In NSCLC stage UICC IIIB, trimodal therapy should be restricted to selected patients only. Standard treatment in these patients is combined. ${ }^{61,63}$

\section{Outcome}

Outcome largely depends on the SVCS etiology. In SCLC, chemotherapy and/or radiotherapy relieved SVCS in $77 \% ; 17 \%$ of those treated had a recurrence of SVCS, as described in a meta-analysis by Rowell and Gleeson. ${ }^{2}$ In NSCLC, $60 \%$ of the patients had relief of SVCS following chemotherapy and/or radiotherapy; however, 19\% of those treated had a recurrence of SVCS. SVC stenting relieved SVCS in $95 \%$. Whereas $11 \%$ of those treated had further SVCS, recanalization was possible in the majority of patients, leading to a long-term patency rate of $92 \%$. Morbidity following stenting was greatest if thrombolytics were administered, which points to an additional danger of thrombolytics.

\section{Summary}

SVCS is often clinically striking, but rarely requires emergency intervention. The majority of cases are due to malignancies. Tissue biopsy is warranted to guide diagnosis and optimize therapy, and is generally safe when performed by experienced practitioners. A multidisciplinary 


\section{Superior Vena Cava Syndrome in Thoracic Malignancies}

approach should be the standard of care for patients with SVCS.

Critical issues at presentation are substantial hemodynamic compromise, substantial laryngeal edema with stridor/threatened airway, and substantial cerebral edema with confusion, obtundation, or stupor. These conditions require immediate action to relieve symptoms promptly and avoid further complications. Interventions should be guided by the objective of not constraining or delaying biopsy. In most cases, biopsies can be taken before intervention, but an exception might be orally anticoagulated patients.

Only patients who require immediate medical intervention due to severe symptoms should receive endovascular stenting for prompt relief of symptoms (recommendation grade 1, evidence grade B). With the advent of stenting, emergency external beam radiation therapy is less used, because it usually does not relieve symptoms for 5-10 days. Radiation treatment should be combined with high doses of corticosteroids to prevent edema formation (grade 2C). In patients with malignancy, after stenting, concurrent radiation therapy with chemotherapy is advised to optimize outcome and prevent tumor growth near the stent, which could cause recurrence of SVCS (tertiary prevention).

In all other cases, if radiological imaging is consistent with malignancy, one should await histological or immunohistochemical diagnosis to start with an optimized therapy. In chemotherapy-naïve or immunotherapy-naïve patients with non-Hodgkin lymphoma, germ-cell tumor, or (possibly) breast cancer, systemic therapy as the sole treatment may be recommended; however, especially for SCLC and NSCLC, systemic therapy concurrent with radiation therapy is the treatment of choice (grade 1C). If symptoms persist, endovascular stenting might bring prompt symptom relief. In patients who have been treated for a malignant cause of SVCS and who suffer from a relapse, endovascular stenting might be a suitable option (grade 2B).

Patients with endovascular stents should receive longterm oral anticoagulation unless there is a contraindication (grade 2C). A reasonable alternative to long-term oral anticoagulation is dual platelet inhibition for 3 months, with aspirin and clopidogrel, always keeping in mind that measures to inhibit coagulation or platelet aggregation might interfere with biopsy. For non-malignant causes of SVCS, causal treatment is usually possible.

SVCS has lost its status as a life-threatening oncologic emergency in most cases. For most treatment options that aim to directly treat the signs and symptoms of SVCS, randomized controlled trials are still lacking.

\section{REFERENCES}

1. Kvale PA, Selecky PA, Prakash UB. Palliative care in lung cancer: ACCP evidence-based clinical practice guidelines (2nd edition). Chest 2007;132(3 Suppl):368S-403S.
2. Rowell NP, Gleeson FV. Steroids, radiotherapy, chemotherapy and stents for superior vena caval obstruction in carcinoma of the bronchus: a systematic review. Clin Oncol (R Coll Radiol) 2002;14(5): 338-351.

3. Wilson P, Bezjak A, Asch M, Barton R, Wong R, Levin W, et al. The difficulties of a randomized study in superior vena caval obstruction. J Thorac Oncol 2007;2(6):514-519.

4. Wilson LD, Detterbeck FC, Yahalom J. Clinical practice. Superior vena cava syndrome with malignant causes. N Engl J Med 2007 3;356(18):1862-1869.

5. Kim HJ, Kim HS, Chung SH. CT diagnosis of superior vena cava syndrome: importance of collateral vessels. AJR 1993;161(3):539542.

6. Trigaux JP, Van BB. Thoracic collateral venous channels: normal and pathologic CT findings. J Comput Assist Tomogr 1990;14(5): 769-773.

7. Chen JC, Bongard F, Klein SR. A contemporary perspective on superior vena cava syndrome. Am J Surg 1990;160(2):207-211.

8. Parish JM, Marschke RF Jr, Dines DE, Lee RE. Etiologic considerations in superior vena cava syndrome. Mayo Clin Proc 1981;56(7): 407-413.

9. Rice TW, Rodriguez RM, Light RW. The superior vena cava syndrome: clinical characteristics and evolving etiology. Medicine (Baltimore) 2006;85(1):37-42.

10. Hunter W. History of aneurysm of the aorta with some remarks on aneurysm in general. Med Obser Inq 1757;1:323-357.

11. McIntire FT, Sykes EM Jr. Obstruction of the superior vena cava; a review of the literature and report of two personal cases. Ann Intern Med 1949;30(5):925-960.

12. Schechter MM. The superior vena cava syndrome. Am J Med Sci 1954;227(1):46-56.

13. Schraufnagel DE, Hill R, Leech JA, Pare JA. Superior vena caval obstruction. Is it a medical emergency? Am J Med 1981;70(6):11691174.

14. Yellin A, Rosen A, Reichert N, Lieberman Y. Superior vena cava syndrome. The myth-the facts. Am Rev Respir Dis 1990;141(5 Pt 1):1114-1118.

15. Rozmus G, Daubert JP, Huang DT, Rosero S, Hall B, Francis C. Venous thrombosis and stenosis after implantation of pacemakers and defibrillators. J Interv Card Electrophysiol 2005;13(1):9-19.

16. Otten TR, Stein PD, Patel KC, Mustafa S, Silbergleit A. Thromboembolic disease involving the superior vena cava and brachiocephalic veins. Chest 2003;123(3):809-812.

17. Sivaram CA, Craven P, Chandrasekaran K. Transesophageal echocardiography during removal of central venous catheter associated with thrombus in superior vena cava. Am J Card Imaging 1996; 10(4):266-269.

18. Van Putten JW, Schlosser NJ, Vujaskovic Z, Leest AH, Groen HJ. Superior vena cava obstruction caused by radiation induced venous fibrosis. Thorax 2000;55(3):245-246.

19. Markman M. Diagnosis and management of superior vena cava syndrome. Cleve Clin J Med 1999;66(1):59-61.

20. Lazzarino M, Orlandi E, Paulli M, Boveri E, Morra E, Brusamolino E, et al. Primary mediastinal B-cell lymphoma with sclerosis: an aggressive tumor with distinctive clinical and pathologic features. J Clin Oncol 1993;11(12):2306-2313.

21. Perez-Soler R, McLaughlin P, Velasquez WS, Hagemeister FB, Zornoza J, Manning JT, et al. Clinical features and results of management of superior vena cava syndrome secondary to lymphoma. J Clin Oncol 1984;2(4):260-266.

22. Gonzalez-Fajardo JA, Garcia-Yuste M, Florez S, Ramos G, Alvarez T, Coca JM. Hemodynamic and cerebral repercussions arising from surgical interruption of the superior vena cava. Experimental model. J Thorac Cardiovasc Surg 1994;107(4):1044-1049 


\section{Superior Vena Cava Syndrome in Thoracic Malignancies}

23. Kishi K, Sonomura T, Mitsuzane K, Nishida N, Yang RJ, Sato M, et al. Self-expandable metallic stent therapy for superior vena cava syndrome: clinical observations. Radiology 1993;189(2):531-535.

24. Mineo TC, Ambrogi V, Nofroni I, Pistolese C. Mediastinoscopy in superior vena cava obstruction: analysis of 80 consecutive patients. Ann Thorac Surg 1999;68(1):223-226.

25. Yu JB, Wilson LD, Detterbeck FC. Superior vena cava syndrome-a proposed classification system and algorithm for management. J Thorac Oncol 2008;3(8):811-814.

26. Stanford W, Doty DB. The role of venography and surgery in the management of patients with superior vena cava obstruction. Ann Thorac Surg. 1986;41(2):158-163.

27. Hetzel M, Arslandemir C, König HH, Buck AK, Nüssle K, Glatting $\mathrm{G}$, et al. F-18 NaF PET for detection of bone metastases in lung cancer: accuracy, cost-effectiveness, and impact on patient management. J Bone Miner Res 2003;18(12):2206-2214.

28. Schumann C, Hetzel J, Babiak AJ, Merk T, Wibmer T, Möller P, et al. Cryoprobe biopsy increases the diagnostic yield in endobronchial tumor lesions. J Thorac Cardiovasc Surg 2010;140(2):417-420.

29. Leschber G, Holinka G, Linder A. Video-assisted mediastinoscopic lymphadenectomy (VAMLA)-a method for systematic mediastinal lymphnode dissection. Eur J Cardiothorac Surg 2003;24(2):192-195.

30. Herth FJ, Eberhardt R, Vilmann P, Krasnik M, Ernst A. Real-time endobronchial ultrasound guided transbronchial needle aspiration for sampling mediastinal lymph nodes. Thorax 2006;61(9):795-798.

31. Schraufnagel DE, Hill R, Leech JA, Pare JA. Superior vena caval obstruction. Is it a medical emergency? Am J Med 1981;70(6):11691174.

32. Furui S, Sawada S, Kuramoto K, Inoue $Y$, Irie $T$, Makita $\mathrm{K}$, et al. Gianturco stent placement in malignant caval obstruction: analysis of factors for predicting the outcome. Radiology 1995;195(1):147-152.

33. Dyet JF, Nicholson AA, Cook AM. The use of the Wallstent endovascular prosthesis in the treatment of malignant obstruction of the superior vena cava. Clin Radiol 1993;48(6):381-385.

34. Nicholson AA, Ettles DF, Arnold A, Greenstone M, Dyet JF. Treatment of malignant superior vena cava obstruction: metal stents or radiation therapy. JVIR1997; 8(5):781-788.

35. Urruticoechea A, Mesia R, Dominguez J, Falo C, Escalante E, Montes A, et al. Treatment of malignant superior vena cava syndrome by endovascular stent insertion. Experience on 52 patients with lung cancer. Lung Cancer 2004;43(2):209-214

36. de Gregorio Ariza MA, Gamboa P, Gimeno MJ, Alfonso E, Mainar A, Medrano J, et al. Percutaneous treatment of superior vena cava syndrome using metallic stents. Eur Radiol 2003;13(4):853-862.

37. Dinkel HP, Mettke B, Schmid F, Baumgartner I, Triller J, Do DD. Endovascular treatment of malignant superior vena cava syndrome: is bilateral wallstent placement superior to unilateral placement? J Endovasc Ther 2003;10(4):788-797.

38. Lanciego C, Pangua C, Chacón JI, Velasco J, Boy RC, Viana A, et al. Endovascular stenting as the first step in the overall management of malignant superior vena cava syndrome. AJR Am J Roentgenol 2009;193(2):549-558

39. Nguyen NP, Borok TL, Welsh J, Vinh-Hung V. Safety and effectiveness of vascular endoprosthesis for malignant superior vena cava syndrome. Thorax 2009;64(2):174-178.

40. Cumming MJ. Superior vena cava syndrome, http://emedicine. medscape.com/article/423504-overview. eMedicine from WebMD. Accessed March 10, 2011

41. Schmucking M, Wendt TG. Tertiärprävention von malignen Tumoren. Onkologe 1998;4(8):747-756. Article in German.

42. Aupérin A, Le Péchoux C, Rolland E, Curran WJ, Furuse K, Fournel $\mathrm{P}$, et al. Meta-analysis of concomitant versus sequential radiochemotherapy in locally advanced non-small-cell lung cancer. J Clin Oncol 2010;28(13):2181-2190.
43. Albain KS, Crowley JJ, Turrisi AT 3rd, Gandara DR, Farrar WB, Clark JI, et al. Concurrent cisplatin, etoposide, and chest radiotherapy in pathologic stage IIIB non-small-cell lung cancer: a Southwest Oncology Group phase II study, SWOG 9019. J Clin Oncol 2002; 20(16):3454-3460.

44. Albain KS, Swann RS, Rusch VW, Turrisi AT 3rd, Shepherd FA, Smith C, et al. Radiotherapy plus chemotherapy with or without surgical resection for stage III non-small-cell lung cancer: a phase III randomised controlled trial. Lancet 2009;374(9687):379-386.

45. Vokes EE, Herndon JE, Kelley MJ et al. Induction chemotherapy followed by chemoradiotherapy compared with chemoradiotherapy alone for regionally advanced unresectable stage III Non-small-cell lung cancer: Cancer and Leukemia Group B. J Clin Oncol 2007; 25(13):1698-1704

46. Tannock IF. Potential for therapeutic gain from combined-modality treatment. Front Radiat Ther Oncol 1992;26:1-15.

47. Peters LJ, Withers HR. Applying radiobiological principles to combined modality treatment of head and neck cancer-the time factor. Int J Radiat Oncol Biol Phys 1997;39(4):831-836.

48. Lonardi F, Gioga G, Agus G, Coeli M, Campostrini F. Double-flash, large-fraction radiation therapy as palliative treatment of malignant superior vena cava syndrome in the elderly. Support Care Cancer 2002;10(2):156-160

49. Egelmeers A, Goor C, van MJ, van den Weyngaert D, Scalliet P. Palliative effectiveness of radiation therapy in the treatment of superior vena cava syndrome. Bull Cancer Radiother 1996;83(3):153157

50. Mose S, Stabik C, Eberlein K, Ramm U, Bottcher HD, Budischewski K. Retrospective analysis of the superior vena cava syndrome in irradiated cancer patients. Anticancer Res 2006;26(6C): 4933-4936.

51. Chan RH, Dar AR, Yu E, Stitt LW, Whiston F, Truong P, et al Superior vena cava obstruction in small-cell lung cancer. Int J Radiat Oncol Biol Phys 1997;38(3):513-520

52. Sculier JP, Evans WK, Feld R, DeBoer G, Payne DG, Shepherd FA, et al. Superior vena caval obstruction syndrome in small cell lung cancer. Cancer 1986;57(4):847-851.

53. Jatoi A, Schild SE, Foster N, Henning GT, Dornfield KJ, Flynn PJ, et al. A phase II study of cetuximab and radiation in elderly and/or poor performance status patients with locally advanced non-smallcell lung cancer (N0422). Ann Oncol 2010;21(10):2040-2044

54. McDermott U, Settleman J. Personalized cancer therapy with selective kinase inhibitors: an emerging paradigm in medical oncology. J Clin Oncol 2009;27(33):5650-5659.

55. Uberoi R. Quality assurance guidelines for superior vena cava stenting in malignant disease. Cardiovasc Intervent Radiol 2006;29(3) 319-322.

56. García Mónaco R, Bertoni H, Pallota G, Lastiri R, Varela M, Beveraggi EM, Vassallo BC. Use of self-expanding vascular endoprostheses in superior vena cava syndrome. Eur J Cardiothorac Surg 2003;24(2):208-211

57. Greillier L, Barlési F, Doddoli C, Durieux O, Torre JP, Gimenez C, Kleisbauer JP. Vascular stenting for palliation of superior vena cava obstruction in non-small-cell lung cancer patients: a future standard procedure? Respiration 2004;71(2):178-183.

58. Lee AY, Levine MN, Baker RI, Bowden C, Kakkar AK, Prins M, et al. Low-molecular-weight heparin versus a coumarin for the prevention of recurrent venous thromboembolism in patients with cancer. N Engl J Med 2003;349(2):146-153.

59. Shimizu N, Moriyama S, Aoe M, Nakata M, Ando A, Teramoto S. The surgical treatment of invasive thymoma. Resection with vascular reconstruction. J Thorac Cardiovasc Surg 1992;103(3):414-420.

60. Charokopos N, Antonitsis P, Klimatsidas M, Giavroglou C, Hatzibaloglou A, Papakonstantinou C. Secondary endovascular repair of a 


\section{Superior Vena Cava Syndrome in Thoracic Malignancies}

reconstructed superior vena cava in a patient with a malignant thymic epithelial neoplasm. Thorac Cardiovasc Surg 2007;55(4):267270.

61. Garrido P, González-Larriba JL, Insa A, Provencio M, Torres A, Isla $\mathrm{D}$, et al. Long-term survival associated with complete resection after induction chemotherapy in stage IIIA (N2) and IIIB (T4N0-1) non small-cell lung cancer patients: the Spanish Lung Cancer Group Trial 9901. J Clin Oncol 2007;25(30):4736-4742.
62. Li J, Dai CH, Shi SB, Chen P, Yu LC, Wu JR. Prognostic factors and long term results of neo adjuvant therapy followed by surgery in stage IIIA N2 non-small cell lung cancer patients. Ann Thorac Med 2009;4(4):201-207.

63. Stupp R, Mayer M, Kann R, Weder W, Zouhair A, Betticher DC, et al. Neoadjuvant chemotherapy and radiotherapy followed by surgery in selected patients with stage IIIB non-small-cell lung cancer: a multicentre phase II trial. Lancet Oncol 2009;10(8):785-793. 